\title{
Jogo educacional sobre avaliação em fisioterapia: uma nova abordagem acadêmica
}

\author{
Educational game on physiotherapy's evaluation: a new academic approach
}

Miriam Weintraub ${ }^{1}$, Philippe Hawlitschek ${ }^{2}$, Silvia Maria Amado João ${ }^{3}$

Estudo desenvolvido no Laboratório de Avaliação Musculoesquelética do Departamento de Fonoaudiologia, Fisioterapia e Terapia Ocupacional da Faculdade de Medicina da Universidade de São Paulo (USP) São Paulo (SP), Brasil.

${ }^{1}$ Fisioterapeuta graduada pelo Curso de Fisioterapia da Faculdade de Medicina da USP São Paulo (SP), Brasil.

${ }^{2}$ Médico graduado pelo Curso de Medicina da Faculdade de Medicina da USP - São Paulo (SP), Brasil.

${ }^{3}$ Docente do Curso de Fisioterapia da Faculdade de Medicina da USP, São Paulo (SP), Brasil.

\section{ENDEREÇO PARA \\ CORRESPONDÊNCIA}

Miriam Weintraub - Rua Morás, 696, apto. 51 - Vila Madalena - CEP: 05434-020 - São Paulo (SP), Brasil-E-mail: miriam@ weintraub.com.br

APRESENTACC̃̃O

fev. 2011

ACEITO PARA PUBLICAÇ̃̃O jul. 2011

FONTE DE FINANCIAMENTO nenhuma

CONFLITO DE INTERESSES nada a declarar

APRESENTAÇ̃̃O EM EVENTOS CIENTÍFICOS

XVIII Congresso Brasileiro de Fisioterapia, em outubro de 2009, no Rio de Janeiro, e na VI Jornadá Acadêmica do Departamento de Fisioterapia, Fonoaudiologia e Terapia Ocupacional da Faculdade de Medicina da USP (Prêmio Profa. Dra. Odete de Fátima Sallas Durigon), em dezembro de 2009.
RESUMO: Este artigo relata a criação de um jogo educacional sobre avaliação em fisioterapia. Uma nova abordagem acadêmica foi proposta para complementar a metodologia atual, basicamente expositiva, especialmente em cursos com conteúdo prático, como a fisioterapia. Uma estratégia pedagógica com casos clínicos e recursos tecnológicos inovadores pode atrair a atenção do estudante, permitindo um aprendizado mais intenso e ativo. O aplicativo foi criado no Adobe Flash CS3 com a linguagem Extensible Markup Language (XML) para a organização das perguntas múltipla escolha. O jogo é composto por quatro salas de atendimento sobre avaliação neurológica, respiratória, musculoesquelética e em terapia intensiva. Há, em cada sala, um caso clínico, seguido de questões múltipla escolha e, por fim, uma questão especial, contendo vídeos ou imagens. Ao responder as questões de forma equivocada, o participante é direcionado a uma sala de estudos com a literatura relacionada ao tema e alguns artigos sugeridos. Para avaliar o impacto desse jogo no processo de aprendizado, serão necessários mais estudos, porém o desenvolvimento de novas estratégias para aprimorar o método de ensino atual é de extrema importância.

Descritores: tecnologia educacional; fisioterapia (especialidade); ensino.

ABSTRACT: This article reports the creation of an education game about physiotherapy's evaluation. It proposes the use of new academic approach to improve the existing teaching method of expositive classes and especially for practical courses like physiotherapy. A pedagogical approach with clinical cases and technological resources is capable of attracting the student attention and may stimulate active learning processes. The game was created with Adobe Flash CS3 combined with an Extensible Markup Language (XML) for the questions. Four treatment rooms were created in four different areas: Neurology, Respiratory, Orthopedic and Intensive Care Unit (ICU). In every room there is a case, followed by multiple choice questions and special questions with pictures and videos. There is also a classroom for each case, where some literature and articles for further studies are suggested. To measure the influence of this educational game in the learning process, more studies are needed, however the development of new strategies to improve the existing teaching method is very important.

Keywords: educational technology; physical therapy (specialty); teaching. 


\section{INTRODUCÃO}

O ensino é uma questão de grande interesse, que passa, atualmente, por uma fase de reestruturação e reorganização, uma vez que se tem à disposição novos meios de comunicação e novas formas de atingir o estudante ${ }^{1}$. Existem ferramentas que podem melhorar a interação professor-aluno e facilitar o processo de aprendizagem se utilizadas de forma adequada.

De acordo com Vasconcellos², a metodologia de ensino atual é considerada expositiva e tem três etapas principais: apresentação do tema, resolução de exercícios-modelo e proposição de exercícios para os alunos. Este modelo evidencia um problema muito importante, que é a não aprendizagem, pois há baixa interação entre o aluno e o objeto estudado, pouca promoção de reflexão e problematização e, por fim, a formação de um aluno passivo e pouco crítico.

Para o aprendizado mais eficaz é necessário mobilizar o aluno para o conhecimento, levar à construção ativa desse conhecimento e propiciar a sua síntese.

Instituições de ensino superior já perceberam a importância de estimular a criação de novos métodos de ensino que diferem do modo tradicional de aulas expositivas, não para substituí-lo, mas para complementar o modelo já existente. Esta iniciativa é de extrema importância para cursos com conteúdos práticos como os da área da saúde, neste caso, em especial, o de Fisioterapia.

Certamente, é um desafio para os professores transmitir o ensinamento prático em aulas expositivas e com carga horária limitada. É também um desafio para os alunos aprender, fixar e aplicar os conceitos transmitidos. Assim, a busca por métodos mais eficientes e atrativos se torna um dever da comunidade acadêmica.

$\operatorname{Varga}^{3}$ et al., Saliba et al. ${ }^{4}$ e Abensur et al. ${ }^{5}$ apresentaram relatos de casos nos quais alguns métodos pouco tradicionais, como discussão de casos clínicos pela internet e simulações da prática profissional, foram utilizados para estimular o processo de ensino-aprendizagem na área da saúde.
Nesta busca, é imprescindível o uso de ferramentas educacionais das quais os estudantes têm afinidade. Haddad et al. ${ }^{6}$ mostram que $83 \%$ dos formandos em fisioterapia no Brasil utilizam sempre ou frequentemente o microcomputador e que a internet é o segundo meio mais usado para atualização, perdendo apenas para a televisão. Recursos amplamente difundidos, como computador e internet, devem ser aproveitados para o crescimento acadêmico e não apenas para lazer.

Um estudo do Centro de Pesquisa Pew, com sede em Washington, revelou que mais da metade dos americanos maiores de 18 anos jogam videogames, sendo que, dos entrevistados com idade universitária (de 18 a 29 anos), 81\% admitiram utilizar jogos online e/ou videogames ${ }^{7}$.

\section{Jogos educacionais}

A inclusão de recursos eletrônicos para aprimorar os modelos de ensino é muito vantajosa ao sistema educacional, pois os estudantes podem acessar um material de apoio com linguagem dinâmica e atrativa, que complementa os materiais já existentes.

Vários autores ${ }^{8-10}$ defendem que o uso de jogos nos modelos pedagógicos pode beneficiar o processo de aprendizagem por motivar o estudante a se adaptar a diversas situações, desenvolvendo raciocínio flexível.

Um jogo educacional deve, porém, seguir algumas diretrizes básicas para alcançar o sucesso esperado. Primeiramente, precisa ser interativo: o jogador deve tomar decisões e ter influencia sobre o rumo do jogo. Um vídeo explicativo ou um texto apresentado com recursos virtuais não pode ser considerado jogo ${ }^{11}$. Ele deve contar também com recompensas a escolhas corretas e bem sucedidas e um enredo coerente.

O objetivo do jogo deve ser a fixação de conceitos e o aprendizado dinâmico, que serão facilitados pelo uso de imagens, vídeos, animações e atividades, atraindo a atenção do jogador. Para que o conhecimento transmitido seja significativo ao receptor, é importante conhecer a realidade na qual o aluno se encontra. Por isso a criação de jogos educacionais também deve ser explorada para incrementar o processo de aprendizagem. A elaboração dos jogos por estudantes é muito defendida por Prensky ${ }^{12}$, que expõe a importância do criador e do usuário pensarem de forma semelhante e estarem em uma situação e um ambiente parecido.

\section{Avaliação em fisioterapia}

$\mathrm{Na}$ fisioterapia acredita-se que "somente através de uma avaliação completa e sistemática é possível se estabelecer um diagnóstico correto" ${ }^{13}$. A avaliação é a chave do tratamento fisioterapêutico. Durante ela são estabelecidos o diagnóstico, o prognóstico, os objetivos do tratamento e a conduta adequada ${ }^{14}$.

Muitas vezes os estudantes têm dificuldade de entender ou memorizar as etapas da avaliação. O tempo de prática, geralmente, é restrito e a disponibilidade de pacientes e supervisores é limitada.

A partir destes fatos foi criado um jogo de avaliação em fisioterapia com o objetivo de permitir aos estudantes de fisioterapia e fisioterapeutas formados a revisão do conteúdo teórico e prático de uma forma que facilite o entendimento e o aprendizado dos métodos de avaliação. Com a utilização do jogo, fica mais fácil a transição do aprendizado teórico para o prático, sendo que os estudantes estarão mais preparados para atender um paciente real no futuro se seu pensamento crítico e a resolução de casos-problemas forem estimulados previamente.

O jogo é abrangente e engloba diversas áreas da fisioterapia, como neurologia, respiratória e músculo-esquelética. Foram desenvolvidas situações cotidianas, desafiando o participante com questões pertinentes de cada área. Para complementar, foram adicionados textos explicativos em uma linguagem simples e objetiva sobre os temas abrangidos.

\section{METODOLOGIA}

O jogo foi estruturado pelos autores no Adobe Flash, um programa de criação de animações e aplicativos interativos de extensão Shockwave Flash File (swf), que funciona em navegadores da internet. Priorizou-se a programação em linguagem ActionScript com imagens em 
formato Joint Photographic Experts Group (.jpeg) e vídeos em Flash Video (.flv). A Extensible Markup Language (XML) foi escolhida como plataforma para a criação de questões múltipla escolha por permitir a organização hierárquica das informações e por ser compatível com o programa de criação.

As estruturas anatômicas em 3D foram criadas no programa $3 \mathrm{~d}$ Max por um especialista da área de criação visual 3D, de acordo com imagens de atlas de anatomia ${ }^{15,16}$, sendo posteriormente tranformadas em vídeos compatíveis com o Adobe Flash.

O roteiro do jogo foi baseado na literatura de avaliação em fisioterapia e em casos clínicos com diagnósticos recorrentes no estágio de fisioterapia do quarto ano da Universidade de São Paulo (USP) e que apresentam prevalência alta na população geral.

Abordou-se distrofia muscular de Duchenne, considerada a distrofia mais comum em crianças ${ }^{17}$; doença pulmonar obstrutiva crônica, que afeta entre 7,8 a 19,7\% da população da América Latina ${ }^{18}$; lesão do ligamento cruzado anterior, com aproximadamente 200 mil novos casos por ano nos Estados Unidos ${ }^{19}$; osteoartrose de joelho, considerada a maior causa de incapacidade física na América do Norte $^{20}$ e acidente vascular encefálico isquêmico, uma das causas mais comuns de morte no mundo ${ }^{21}$.

Os casos clínicos apresentados no jogo são fictícios e representam um paciente com características típicas das patologias selecionadas.

As questões múltipla escolha foram formuladas pela autora do projeto e apresentadas aos supervisores do curso de fisioterapia da USP para correção e adequação do conteúdo. Os vídeos e as questões diferenciadas foram criados com base em três referências principais ${ }^{22-24}$.

\section{RESULTADOS}

O jogo é composto de quatro "salas de atendimento" (Figura 1) divididas em avaliação musculoesquelética, respiratória, neurológica e, por fim, uma que abrange as três áreas de conhecimento. Em cada uma delas o participante tem acesso à história do paciente e a alguns exames complementares antes de ser confrontado com perguntas sobre a avaliação, os objetivos do tratamento e a conduta adequada. Quando a resposta escolhida for incorreta, o participante passa por uma etapa de revisão teórica do tema nas "salas de estudos", onde encontra referências bibliográficas, textos, imagens e vídeos explicativos.

Na primeira sala, o caso clínico apresentado é sobre um paciente hipotético de neurologia infantil, com diagnóstico médico de distrofia muscular de Duchenne e queixa funcional de dificuldade para deambular (Figura 2). Nas questões iniciais o participante é questionado sobre o desenvolvimento neuro-psico-motor e sobre os reflexos primitivos (Figura 3). Seguem questões sobre as distrofias musculares em geral e sobre a distrofia muscular de Duchenne. Por fim, temos uma questão com três colunas distintas. Uma com o nome, outra com a descrição e a última com vídeos demonstrativos de tipos clássicos de marcha. Sendo assim, o jogador deve ligar as três colunas corretamente (Figura 4).

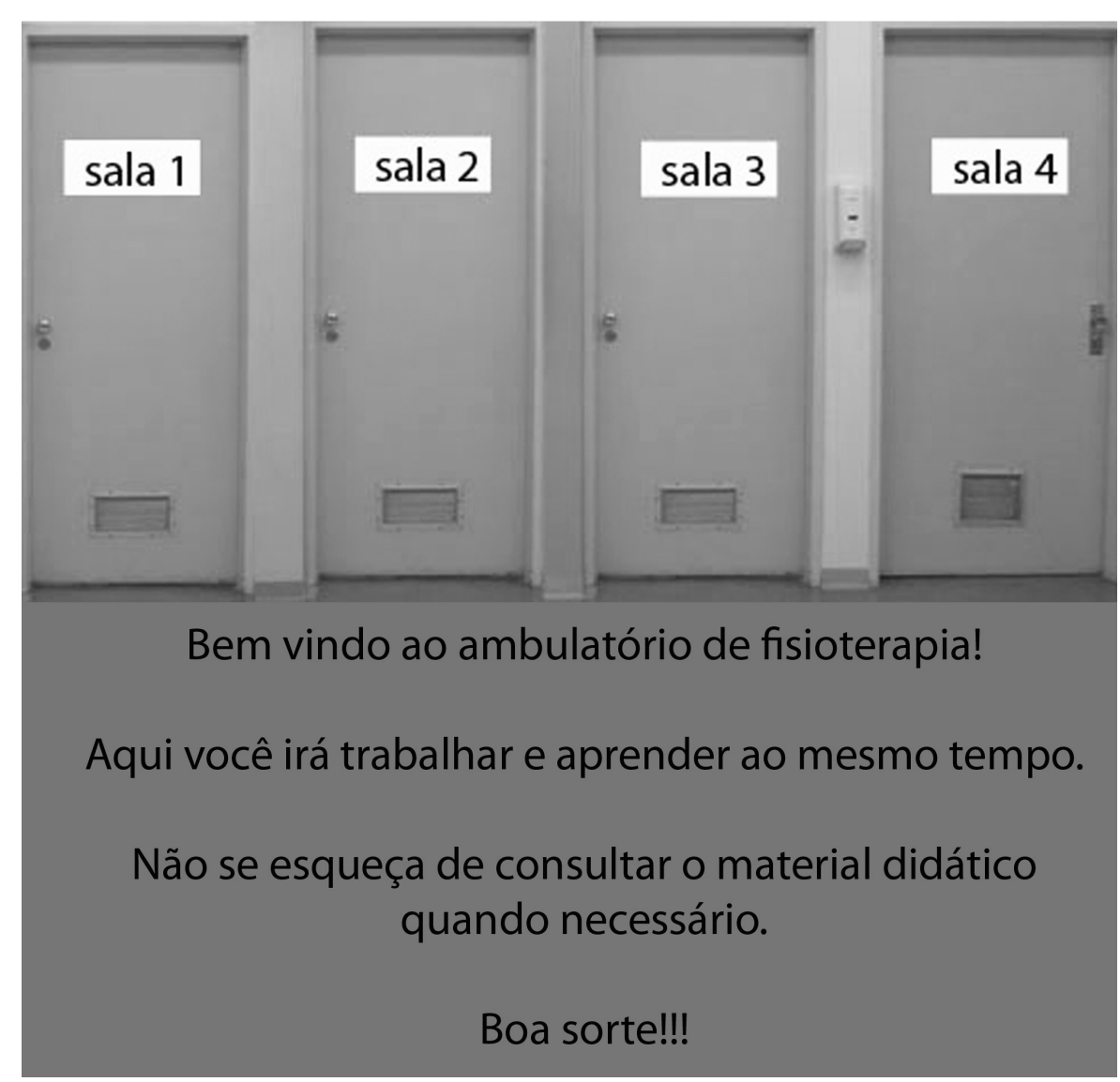

Figura 1. Introdução às "salas de atendimento"
A parte teórica desta sala contém uma literatura direcionada à neurologia infantil e à avaliação fisioterápica em neurologia ${ }^{24-27}$ (Figura 5).

Na sala 2, de avaliação respiratória, há um caso de um paciente com doença pulmonar obstrutiva crônica (DPOC) com queixa de dispneia. O participante é questionado sobre a avaliação do paciente, incluindo a ausculta pulmonar e a palpação da caixa torácica e também sobre a indicação de oxigenoterapia, os objetivos com um paciente DPOC e a conduta adequada. A última questão contém radiografias de tórax, sendo que o participante deve reconhecer algumas características específicas de cada imagem, como pneumotórax e abscessos pulmonares. Na sala de estudos é apresentada literatura sugerida e resumo teórico sobre radiografias de tórax e espirometria $^{25,28}$.

O caso clínico da sala de avaliação musculoesquelética é referente à lesão do ligamento cruzado anterior de joelho. As questões de múltipla escolha abordam temas como a função da patela e 
da musculatura da coxa, a localização dos ligamentos, os testes clínicos especiais e conduta ideal no pós-operatório de reconstrução do ligamento cruzado anterior. Há duas questões especiais nas quais o participante deve escrever o músculo, sendo testado no teste de força de cada vídeo. Na etapa teórica dessa sala, temos à disposição referências específicas e um joelho em 3D que demonstra em detalhes a flexão e a extensão do joelho ${ }^{13,22,25,29-34}$.

Na última sala, o paciente é confrontado com um paciente na Unidade de Terapia Intensiva (UTI), entubado após um acidente vascular encefálico, com antecedente de osteoatrose de joelho. As questões abordam temas como gasometria, ventilação mecânica, quadro clínico pós-acidente vascular encefálico, avaliação de tônus, conduta na osteoatrose e consequências do imobilismo. A questão diferencial desta sala exige que o participante identifique que nível medular é testado em cada reflexo realizado nas avaliações dos pacientes. $\mathrm{Na}$ "sala de estudos" temos algumas informações importantes sobre ventilação mecânica, sobre a diferença de um sistema osteomuscular saudável e outro com osteoatrose e as causas principais de um acidente vascular hemorrágico e isquêmico ${ }^{35-40}$.

\section{DISCUSSÃO}

A inclusão de jogos educacionais no ensino é uma nova estratégia para mobilizar o aluno a adquirir o conhecimento objetivado e atingir um nível mais profundo de aprendizado, no qual além de memorizar o conteúdo teórico, deve ser capaz de relacionar, refletir e aplicar os novos conceitos em situações práticas $^{2}$.

O jogo permite a inserção em um ambiente propício ao aprendizado, pois os estudantes estão ativamente engajados a uma tarefa direcionada e conseguem explorar múltiplas capacidades ${ }^{41}$.

Como o jogo é criado por alunos para alunos, o ambiente e a realidade do futuro jogador são conhecidos com clareza, o criador e o receptor dividem as mesmas experiências, expectativas e interesses e, portanto, há maior possibilidade do conhecimento se tornar significativo e interessante.

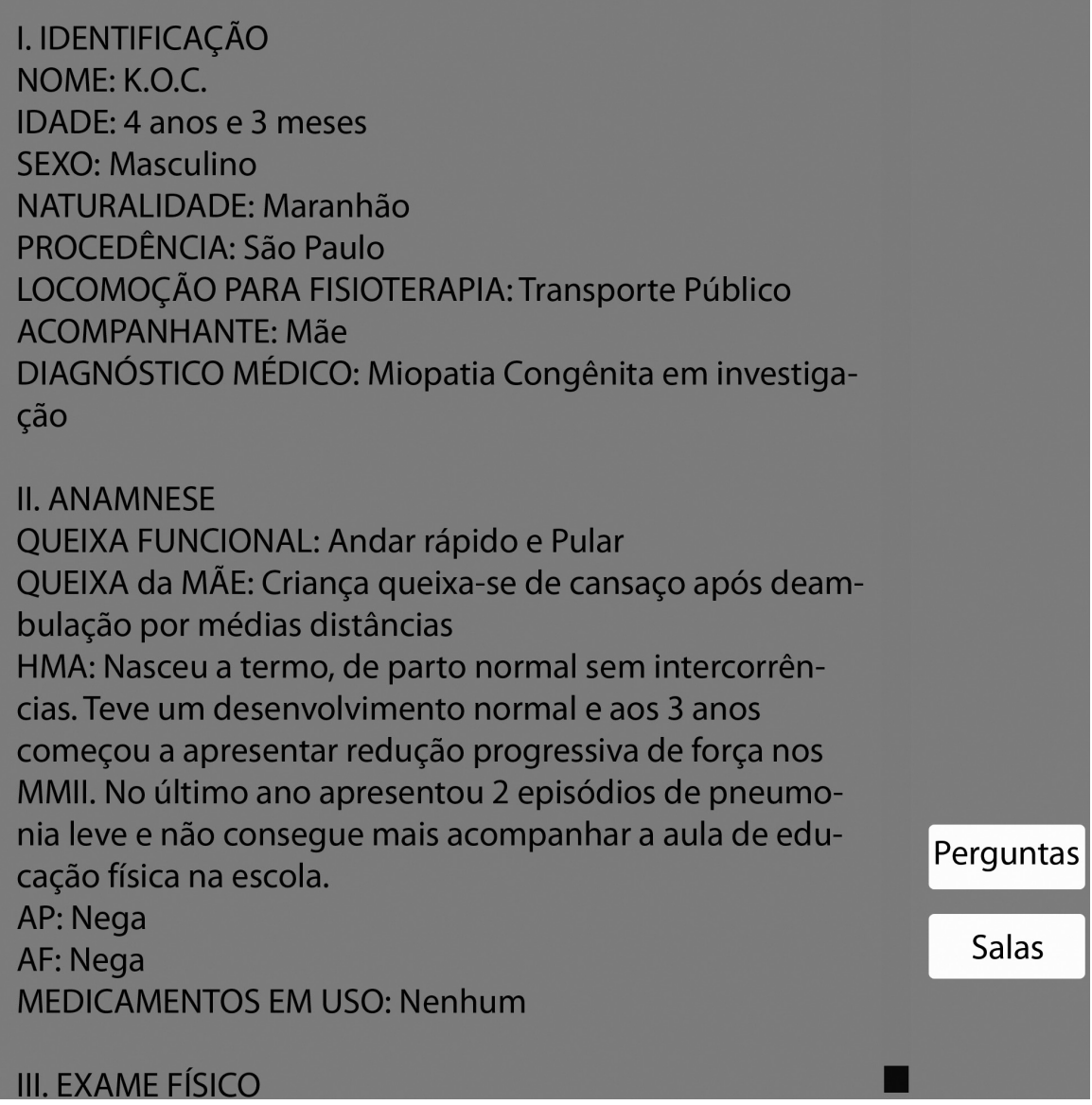

Figura 2. Caso clínico da sala 1

\section{Sobre o desenvolvimento normal :}

\section{No primeiro mês a criança permanece num padrão extensor}

No terceiro mês a criança não extende a cabeça no decúbito ventral

No sétimo mês a criança passa da sedestação para bipedestação

No décimo mês a criança ngatinha com rapidez

\section{Com um ano a criança corre e chuta uma bola}

\section{História}

Próxima

Salas

Figura 3. Questão de múltipla escolha da sala 1 
Os jogos também podem preparar o aluno para a última etapa do processo de aprendizagem, que é o contato direto com o paciente e a aplicação na prática clínica dos conhecimentos obtidos ${ }^{2}$.
Atualmente podemos considerar o aluno universitário mais maduro em seu processo de aprendizado, com maior disposição para aulas direcionadas à aplicação da teoria a problemas

\section{A queixa do paciente é deambulação. Relacione cada marcha, com suas características e vídeo}

\section{A) Marcha em Tesoura}

B) Marcha da Ataxia Sensorial

C) Marcha Hemiplégica

1) Tronco anteriorizado e passos curtos

2) Movimentos bruscos e base aumentada

3) Ligeira flexão de joelhos e adução de quadril

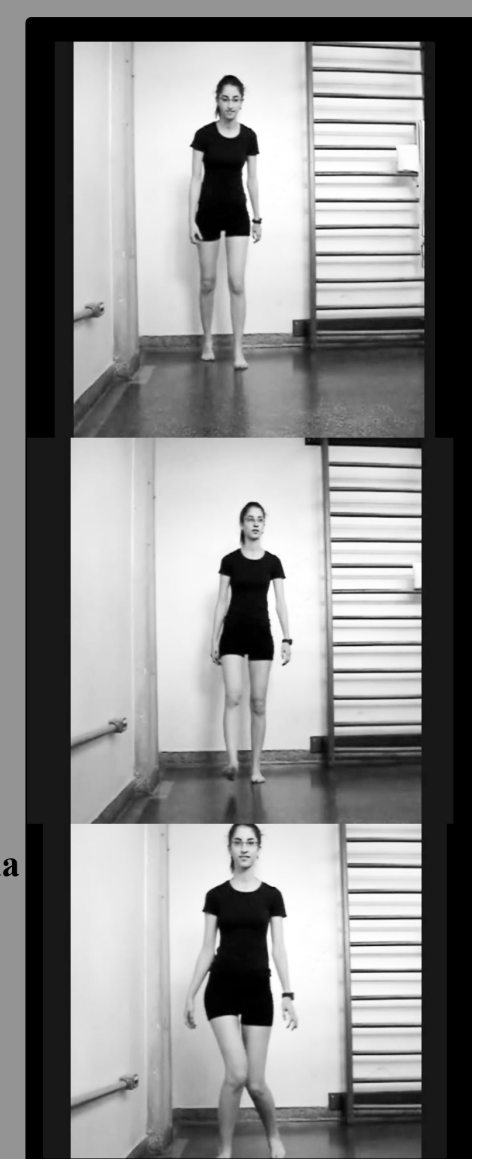

Figura 4. Questão final da sala 1

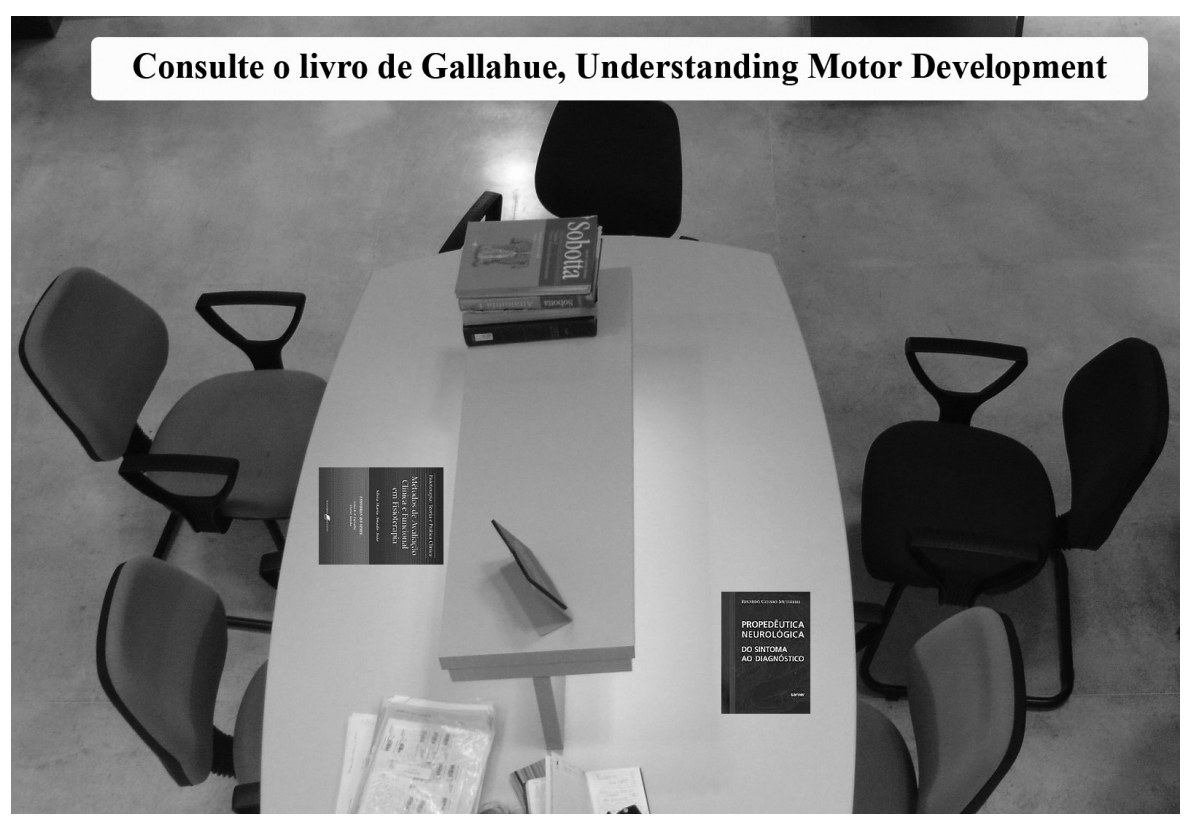

Figura 5. Área de estudos da sala 1 relevantes que serão encontrados em sua jornada profissional. Porém, o modelo adotado ainda é predominantemente expositivo. A retenção do conhecimento passa de $20 \%$ em uma exposição com uso de recursos multimídia para $75 \%$ em uma atividade voltada para a prática ${ }^{42}$, portanto o formato de ensino atual não favorece o aprendizado.

Contudo, é importante ressaltar que a utilização de jogos educacionais não substitui a necessidade do aprendizado prático de contato direto com o paciente e aplicação das técnicas de fisioterapia em seres humanos. O uso de recursos visuais e interativos não se iguala ao atendimento de pacientes reais, mas pode facilitar a transição da sala de aula para a prática clínica.

A criação e aplicação dos jogos como ferramenta de ensino permitem a interação da teoria e da prática, na qual situações reais ou fictícias são simuladas, desafiando a capacidade do aluno de resolver problemas e aplicar o conhecimento ${ }^{43,44}$ adquirido.

Para confirmar que este jogo terá impacto no processo de aprendizagem devemos testar em um modelo científico sua eficácia. Porém, há indícios de que esta estratégia traga resultados satisfatórios pelas excelentes experiências de jogos educacionais do Massachusetts Institute of Technology (MIT), da Oxford e da Universidade Politécnica de Hong Kong, entre outros $^{12,45}$.

A inserção de jogos educacionais no meio acadêmico traz a possibilidade dos estudantes aprenderem com casos clínicos reais ou fictícios ${ }^{46}$, direcionados à prática clínica e ficando mais próximos dos desafios que encontrarão nos atendimentos de fisioterapia.

Este aplicativo, criado a partir de uma base teórica sólida associada a casos clínicos, imagens e vídeos, é uma nova opção para complementar o currículo já existente. A estratégia possui grandes chances de sucesso, considerando os exemplos utilizados em outras áreas acadêmicas. É importante ressaltar que o aplicativo criado não depende de alto investimento financeiro e pode ser utilizado em qualquer computador com acesso à internet. 


\section{CONCLUSÃO}

Este projeto pioneiro na área de fisioterapia mostrou que é possível criar um material acadêmico diferenciado, que pode complementar os métodos tradicionais amplamente utilizados. A introdução de recursos dinâmicos e interativos ao processo de aprendizagem permite que o estudante seja colocado em situações nas quais o pensamento crítico e a capacidade de adaptação sejam estimulados.
No futuro será importante testar a eficiência do jogo no processo de aprendizagem do conteúdo abordado e investir em jogos educacionais com outros objetivos, como avaliação dos estudantes ou material de orientação para pacientes e cuidadores.

\section{REFERÊNCIAS}

1. Cunha MI, Marsico HL, Borges FA, Tavares P. Inovações pedagógicas na formação inicial de professores. In: Fernandes CMB, Grillo M. Educação superior: travessias e atravessamentos. Canoas: Ulbra; 2001. p. 33-90.

2. Vasconcellos CS. Metodologia Dialética em Sala de Aula. AEC. 1995;21(83).

3. Varga CRR, Almeida VC, Germano CMR, Melo DG, Chachá SGF, Souto BGA, et al. Relato de experiência: o uso de simulações no processo de ensino-aprendizagem em medicina. Rev Bras Educ Med. 2009;33(2):291-7.

4. Saliba NA, Moimaz SAS, Chiaratto RA e Tiano AVP. A utilização da metodologia PBL em Odontologia: descortinando novas possibilidades ao processo ensinoaprendizagem. Rev Odonto Ciênc. 2008;23(4):392-6.

5. Abensur SI, Abensur H, Malheiros DMAC, Zatz R e Barros RT. Uso da internet como um ambiente para discussão de casos clínicos. Rev Bras Educ Med. 2007;31(3):291-5.

6. Haddad AE, Pierantoni CR, Ristoff D, Xavier I, Giolo J, Silva LB. A trajetória dos cursos de graduação na área da saúde: 1991-2004. INEP; 2006.

7. France Presse. FolhaOnline. São Paulo [atualizada em 08/12/2008; acesso em 17/08/2009]. Disponível em: http://www1.folha.uol.com.br/folha/informatica/ ult124u476547.shtml

8. Moreno-Ger P, Burgos D, Martínez-Ortiz I, Sierra J, Fernández-Manjón B. Educational game design for online education. Comput Human Behav. 2008;24(6):2530-40.

9. Teixeira JSF, Sá EJV, Fernandes CT. Representação de jogos educacionais a partir do modelo de objetos de aprendizagem. WIE'2007 - Workshop de Informática na Educação, Rio de Janeiro; 2007.

10. Hill JM, Ray CK, Blair JR, Carver CA. Puzzles and games: addressing different learning styles in teaching operating systems concepts. 34th SIGCSE Technical Symposium on Computer Science Education. Reno, Nevada; 2003.

11. Habgood J, Overmars M. The game maker's apprentice: game development for beginners. 1ª ed. Apress; 2006.

12. Prensky M. Students as designers and creators of educational computer games. Who else? Br J Educ Technol. 2007;39(6):1004-19.
13. Magee DJ. Avaliação Musculoesquelética. 4ª ed. São Paulo: Editora Manole; 2002.

14. White J. Avaliação musculoesquelética. Em: O'Sullivan SB, Schmitz TJ. Fisioterapia avaliação e tratamento. $2^{\mathrm{a}}$ ed. São Paulo: Editora Manole; 2004:101-32.

15. Netter FH. Atlas de anatomia humana. $4^{\mathrm{a}} \mathrm{ed}$. Rio de Janeiro: Elsevier; 2008.

16. Putz R, Pabst R. Atlas de anatomia humana Sobotta. $21^{\text {a }}$ ed. Rio de Janeiro: Guanabara Koogan; 2000:Volume II.

17. Ciafaloni E, Fox DJ, Pandya S, Westfield CP, Puzhankara S, Romitti PA, et al. Delayed diagnosis in duchenne muscular dystrophy: data from the muscular dystrophy surveillance, tracking, and research network. J Pediatr. 2009;155(3):380-5.

18. Mannino DM, Braman S. The epidemiology and economics of chronic obstructive pulmonary disease. Proc Am Thorac Soc. 2007;4:502-6.

19. Beynnon BD, Johnson RJ, Abate JA, Fleming BC, Nichols CE. Treatment of anterior cruciate ligament injuries, part I. Am J Sports Med. 2005;33(10):1579-602.

20. Razmjou H, Finkelstein JA, Yee A, Holtby R, Vidmar M, Ford M. Relationship between preoperative patient characteristics and expectations in candidates for total knee arthroplasty. Physiother Can. 2009; 61(1):38-45.

21. Stroebele N, Müller-Riemenschneider F, Nolte $\mathrm{CH}$, Müller-Nordhorn J, Bockelbrink A, Willich SN. Knowledge of risk factors, and warning signs of stroke: a systematic review from a gender perspective. Int J Stroke. 2011;6(1):60-6.

22. Kendall FP, McCreary EK, Provance PG, Rodgers MM, Romani WA. Músculos: provas e funções. $5^{\mathrm{a}} \mathrm{ed}$. São Paulo: Manole; 2007.

23. Victor $M$, Ropper $\mathrm{AH}$. Adams and Victor's principles of neurology. $7^{\mathrm{a}}$ ed. Nova lorque: MacGraw-Hill; 2001.

24. Mutarelli EG. Propedêutica neurológica: do sintoma ao diagnóstico. São Paulo: Sarvier; 2000.

25. Amado-João SM. Métodos de avaliação clínica e funcional em fisioterapia. Rio de Janeiro: Guanabara Koogan; 2006. 


\section{Referências (cont.)}

26. Gallahue DL, Ozmun JC. Understanding motor development: infants, children, adolescents, adults. $6^{\mathrm{a}}$ ed. Nova lorque: McGraw-Hill; 2006.

27. Tecklin JS. Fisioterapia pediátrica. $3^{\mathrm{a}}$ ed. Porto Alegre: Artmed; 2002.

28. Presto B, Damázio L. Fisioterapia respiratória. $4^{\mathrm{a}}$ ed. São Paulo: Elsevier; 2009.

29. Sacco ICN, Tanaka C. Cinesiologia e biomecânica dos complexos articulares. Rio de Janeiro: Guanabara Koogan; 2008.

30. O'Sullivan SB, Schmitz TJ. Fisioterapia: avaliação e tratamento. 4ª ed. São Paulo: Manole; 2004.

31. Marques AP. Manual de goniometria. $2^{\text {a }}$ ed. São Paulo: Manole; 2003.

32. Podesta L, Magnusson J, Gillette T. Reconstrução do ligamento cruzado anterior. In: Maxey L, Magnusson J. Reabilitação pós-cirúrgica para o paciente ortopédico. Rio de Janeiro: Guanabara Koogan; 2003.

33.Shumway-Cook A, Woollacott MH. Controle motor: teoria e aplicações práticas. $2^{a}$ ed. São Paulo: Manole; 2003.

34. Reid DC. Sports injury: assessment and Rrehabilitation. $1^{\text {a }}$ ed. Nova Iorque: Elsevier; 1993.

35. Hunter DJ, Lo GH. The management of osteoarthritis: an overview and call to appropriate conservative treatment. Rheum Dis Clin North Am. 2008;34(3):689-712.

36. Misulis KE, Head TC. Netter Neurologia Essencial. $1^{\text {a }}$ ed. Rio de Janeiro: Elsevier; 2008.
37. Tanaka C, Fu C. Revista Crefito SP. 2008. Ano 5 - $2^{\text {a }}$ ed, jun/jul:24-25.

38. Mikesky AE, Mazzuca SA, Brandt KD, Perkins SM, Damush T, Lane KA. Effects of strength training on the incidence and progression of knee osteoarthritis. Arthritis Rheum. 2006;15;55(5):690-9.

39.Zuñiga QGP. Ventilação mecânica básica. 1ª ed. São Paulo: Atheneu; 2005.

40. Carvalho CRR. Ventilação mecânica, Volume II Avançado. São Paulo: Atheneu; 2000.

41. Smeets E. Does ICT contribute to powerful learning environments in primary education? Comput Educ. 2005;44(3):343-55.

42. Zemke R, Zemke S. 30 things we know for sure about adult learning. Innovation Abstracts. 1984;8(9):1-3.

43. Quinn CN. Engaging learning: designing e-learning simulation games. Pfeiffer, John Wiley and Sons, San Francisco; 2005.

44. Amory A. Game object model version II: a theoretical framework for educational game development. Educ Tech Res Dev. 2007;55:51-77.

45. Wang W. O aprendizado através de jogos para computador: por uma escola mais divertida e mais eficiente. Portal da Família [acesso em 01/2009]. Disponível em: http://portaldafamilia.org/artigos/ artigo479.shtml.

46. Shiroma PR, Massa AA, Alarcon RD. Using game format to teach psychopharmacology to medical students. Med Teach. 2011;33(2):156-60. 